\section{Uso de benzodiazepinas en adultos mayores en América Latina}

\author{
SAÚL GÓMEZ ${ }^{\mathrm{a}}$, TOMÁS LEÓN ${ }^{1}$, MAXIMILIANO MACUER $^{\mathrm{a}}$, \\ MARIANA ALVES ${ }^{2}$, SERGIO RUIZ $^{1,3,4}$
}

\section{Benzodiazepine use in elderly population in Latin America}

Background: Growing information has emphasized the risk of benzodiazepines (BZD), particularly among the elderly. However, the information available in Latin America is scarce. Aim: to review the available information on the use of BZD in older adults in Latin America to achieve an overview of the information currently available, and a thorough understanding of this phenomenon in our region. Methods: A systematic review with MeSH terms "elderly", "latinamerican" and "benzodiazepines" was performed in PubMed and with each Latin American country. A search in databases SciELO and LILACS was also performed. In all, 126 items of finally selected 21 that met the inclusion criteria. Results: Studies show that consumption of benzodiazepines in the elderly population in Latin America is high, with a preponderance of long half-life benzodiazepines in women, and frequent self-medication. Conclusions: The revised articles establish the importance of further study of the phenomenon of the use of benzodiazepines in our elderly population. Strikingly, scientific information is scarce, with most studies coming from only one country (Brazil). Moreover, most of them are transversal and descriptive, with few studies that explore long-term side effects, or specific hypotheses. Further studies should address these important issues.

(Rev Med Chile 2017; 145: 351-359)

Key words: Aged; Benzodiazepines; Frail Elderly; Latin America.
'Departamento de Psiquiatría,

Centro Interdisciplinario de

Neurociencias, Escuela de

Medicina, Pontificia Universidad

Católica de Chile. Santiago, Chile.

${ }^{2}$ Unidade Universitaria de

Geriatría/Medicina Interna,

Hospital Pulido Valente. Lisboa,

Portugal.

${ }^{3}$ Institute of Medical Psychology

and Behavioral Neurobiology,

Universidad de Tuebingen.

Alemania.

${ }^{4}$ Laboratory for Brain-Machine

Interfaces and Neuromodulation,

Pontificia Universidad Católica de

Chile. Santiago, Chile.

aAlumno, Escuela de Medicina,

Pontificia Universidad Católica de

Chile. Santiago, Chile.

Fuente de apoyo financiero:

Dirección de Investigación,

Escuela de Medicina, Pontificia

Universidad Católica de Chile.

Recibido el 12 mayo de 2016 , aceptado el 6 de junio de 2016 .

Correspondencia a:

Dr. Sergio Ruiz Poblete

Departamento de Psiquiatría,

Escuela de Medicina,

Centro Interdisciplinario de

Neurociencias, Pontificia

Universidad Católica de Chile.

Marcoleta 391, oficina 201c.

Santiago, Chile.

sruiz@uc.cl
U tilizadas desde la década de los cincuenta, las benzodiazepinas (BZD) son empleadas ampliamente en la práctica médica ${ }^{1-3}$ gracias a su capacidad de producir ansiolisis, hipnosis, relajación muscular y disminuir el umbral convulsivante ${ }^{1,4-6}$. Sin embargo, su consumo crónico se ha asociado a múltiples efectos secundarios, incluido abuso y dependencia ${ }^{7}$.

En adultos mayores (AM), las BZD deben ser utilizadas con precaución, debido, entre otras razones, a los cambios en la farmacodinamia y la farmacocinética inducidas por la edad y la polifarmacia ${ }^{8}$. En efecto, de acuerdo a los "Criterios de Beers", que clasifican los medica- mentes según la seguridad para su uso en AM, las BZD son categorizadas como drogas no recomendadas 9 . Además de su potencial adictivo, el consumo crónico de BZD en AM se ha asociado a otros efectos secundarios importantes, dentro de los que se encuentran el aumento del riesgo de caídas, disfunciones cognitivas y asociación con el desarrollo de demencia (e.g. Alzheimer's Disease $)^{10-13}$. A pesar de sus riesgos, la literatura sugiere que el uso de BZD en AM es frecuente en-17. $^{14}$.

En Latinoamérica, el uso racional de psicofármacos en AM es de particular relevancia considerando, por un lado, el acelerado crecimiento 
de la población mayor de 60 años, y la situación de fragilidad (económica y social) de esta población $^{18,19}$. Sin embargo, pese a la importancia del uso cuidadoso de BZD en AM, la información detallada sobre el consumo de BZD en este subgrupo etario en nuestra región es escasa, debiéndose con frecuencia extrapolar hallazgos provenientes de otras poblaciones ${ }^{20}$.

El objetivo general de este estudio es realizar una revisión de la información disponible sobre el uso de BZD en AM en Latinoamérica. Los objetivos específicos son revisar la literatura científica desarrollada en Latinoamérica en esta población y explorar la evidencia disponible en relación a las variables sociodemográficas asociadas a su uso, la frecuencia de consumo, la indicación médica por las cuales se utilizan y los efectos adversos más frecuentes. Además, se espera lograr un panorama general de la información ya disponible, y los estudios faltantes para lograr una comprensión cabal de este fenómeno en nuestra región.

\section{Material y Método}

En primer lugar, se realizó una revisión sistemática con los términos MeSH "elderly", "latinamerican" y "benzodiazepines" en PubMed. Además, se realizó una búsqueda en la misma base de datos con términos libres que incluían el nombre de cada uno de los países latinoamericanos asociado a "elderly" y "benzodiazepines". En total, se encontraron 72 artículos en PubMed.

De igual manera se realizó una búsqueda en SciELO, donde se encontraron dos publicaciones; y en LILACS, donde se encontraron 52 trabajos más.

En total, se seleccionaron 126 artículos. Se realizó lectura de todos estos resúmenes, seleccionándose los trabajos que cumplieran con los siguientes criterios de inclusión: a) Lenguaje en español, inglés o portugués; b) Que la población estudiada sea latinoamericana e incluya adultos mayores; c) Corresponder a trabajos originales y no revisiones bibliográficas o meta análisis.

Finalmente, con estos criterios se seleccionaron 37 artículos para su lectura completa, pudiéndose acceder a 31 de ellos. De éstos, en lectura detallada, se eliminaron diez artículos porque no aportaban información específica respecto al consumo de
BZD en AM, por lo que el resultado final incluye 21 estudios.

\section{Resultados}

Los artículos seleccionados en la revisión incluyeron artículos de cinco países latinoamericanos: Argentina (2 trabajos ${ }^{21,22}$, Brasil (14 trabajos ${ }^{17,23-35}$ ), Chile ( 3 trabajos $\left.^{3,36,37}\right)$, Cuba (1 trabajo $^{38}$ ) y México $\left(1\right.$ trabajo $\left.^{39}\right)$. Diecisiete de los 21 estudios incluidos fueron de carácter transversal.

De los 21 estudios seleccionados, 17 fueron publicados en revistas latinoamericanas $^{3,17,21-24,26,27,29-33,35,37-39}$, mientras que los cuatro restantes se encontraban en revistas europeas o norteamericanas ${ }^{25,28,34,36}$. En tres trabajos la muestra de AM estudiada correspondió a población hospitalizada ${ }^{21,27,28}$, en tres estudios a población institucionalizada ${ }^{24,30,37}$, en cuatro trabajos a población ambulatoria de medicina general ${ }^{3,29,35,38}$, en cuatro artículos a población ambulatoria de psiquiatría/psicología ${ }^{22,32,33,39}$, en un estudio a población en servicio de urgencias psiquiátricas ${ }^{34} y$ en seis trabajos a población de la comunidad (no consultante) $)^{17,23,25,26,31,36}$.

La mayoría presenta como objetivo central el estudio de la prevalencia de uso de BZD en AM, y la exploración de sus características demográficas y aspectos generales de salud. Un pequeño porcentaje de estos estudios tuvo como objetivo principal el estudio de patología específica en relación al consumo de BZD (caídas, fracturas, deterioro cognitivo $)^{23,24,26-28,37}$.

El detalle respecto al tipo de población evaluada y el diseño experimental de cada estudio se resume en la Tabla 1.

\section{Frecuencia de uso de BZD en AM}

De los artículos analizados, doce especificaron la frecuencia de uso en personas mayores de 60 años. En general, estos estudios confirman el alto consumo de BZD a nivel latinoamericano. En la población general (no consultante) de AM, se reportó el uso de BZD en $21,7 \%{ }^{17}, 22 \%{ }^{23}, 9,3 \%{ }^{26}$, hasta $49,5 \%{ }^{36}$. En servicios de atención primaria de salud, se reportó una frecuencia de uso que fluctúa entre $25 \%{ }^{29}, 28,1 \%{ }^{22}$ y $61,4 \%{ }^{34}$. En pacientes AM hospitalizados o institucionalizados, la frecuencia de consumo de BZD se reportó en un amplio rango de porcentajes, desde $7,4 \%{ }^{30}, 17,6 \%{ }^{28}, 23,6 \%{ }^{24}$, $38 \%{ }^{37}$, hasta $50,8 \%{ }^{21}$. 
Tabla 1. Estudios que exploran el uso de BZD en adultos mayores en América Latina

\begin{tabular}{|c|c|c|c|}
\hline $\begin{array}{l}\text { Autor, } \\
\text { año }\end{array}$ & País & Tipo de muestra y $n$ del estudio & $\begin{array}{l}\text { Diseño } \\
\text { experimental }\end{array}$ \\
\hline $\begin{array}{l}\text { Marzi, } \\
2013\end{array}$ & Argentina & $\begin{array}{l}\text { Se analizaron registros de } 179 \text { pacientes hospitalizados mayores de } 60 \\
\text { años, en Hospital Geriátrico Provincial de Rosario }\end{array}$ & Transversal \\
\hline $\begin{array}{l}\text { Rojas, } \\
2010\end{array}$ & Argentina & $\begin{array}{l}\text { Se evaluaron } 313 \text { pacientes mayores de } 60 \text { años que consultaron por } \\
\text { trastornos cognitivos al Laboratorio de Memoria del Hospital Municipal } \\
\text { Abel Zubizarreta (136), Hospitales Privados (13) y derivaciones de otros } \\
\text { hospitales municipales (164), durante un año }\end{array}$ & Prospectivo \\
\hline $\begin{array}{l}\text { Alvarenga, } \\
2008\end{array}$ & Brasil & $\begin{array}{l}\text { Se entrevistaron a } 1.606 \text { pacientes con edad mayor o igual a } 60 \text { años de } \\
\text { la ciudad de Bambuí }\end{array}$ & Transversal \\
\hline $\begin{array}{l}\text { Alvarenga, } \\
2009\end{array}$ & Brasil & $\begin{array}{l}\text { De } 1.606 \text { pacientes mayores a } 60 \text { años de la ciudad de Bambuí, se seleccio- } \\
\text { naron } 1.419 \text { (se excluyeron pacientes sin un "proxy respondent" y quienes } \\
\text { rechazaron una toma de muestras de laboratorio) }\end{array}$ & Transversal \\
\hline $\begin{array}{l}\text { Bicca, } \\
2008\end{array}$ & Brasil & $\begin{array}{l}\text { Se estudiaron } 123 \text { mujeres institucionalizadas, con edad mayor o igual a } \\
60 \text { años, divididas en dos grupos según consumo o no de BZD ( } 29 \text { y } 94 \\
\text { pacientes, respectivamente). Se excluyeron pacientes con trastornos psicó- } \\
\text { ticos y depresión severa }\end{array}$ & Transversal \\
\hline $\begin{array}{l}\text { Brunoni, } \\
2013\end{array}$ & Brasil & $\begin{array}{l}\text { Se entrevistaron } 15.105 \text { pacientes como parte del estudio ELSA-Brasil (eda- } \\
\text { des entre 35-74 años), en seis ciudades diferentes de Brasil }\end{array}$ & Transversal \\
\hline $\begin{array}{l}\text { Chaimowicz, } \\
2000\end{array}$ & Brasil & $\begin{array}{l}\text { De } 1.024 \text { familias evaluadas en la vecindad de Arnaldos, se seleccionaron } \\
161 \text { personas mayores de } 65 \text { años }\end{array}$ & Transversal \\
\hline $\begin{array}{l}\text { Coutinho, } \\
2002\end{array}$ & Brasil & $\begin{array}{l}\text { Se estudiaron } 169 \text { casos de pacientes con edad igual o mayor a } 60 \text { años } \\
\text { hospitalizados en cinco hospitales de Río de Janeiro, con fracturas severas } \\
\text { por caídas en los años } 1998 \text { y 1999. Los pacientes controles fueron } 315\end{array}$ & Caso-control \\
\hline $\begin{array}{l}\text { Coutinho, } \\
2008\end{array}$ & Brasil & $\begin{array}{l}\text { Se estudiaron } 250 \text { casos de pacientes hospitalizados mayores de } 60 \text { años, } \\
\text { en cinco hospitales de Brasil con fracturas severas por caidas dentro de } \\
\text { 2002-2003. Se incluyeron además } 250 \text { controles }\end{array}$ & Caso-control \\
\hline $\begin{array}{l}\text { Gorzoni, } \\
2012\end{array}$ & Brasil & $\begin{array}{l}\text { Se estudiaron } 100 \text { registros médicos de pacientes } \mathrm{AM} \text { en primer control } \\
\text { ambulatorio geriátrico }\end{array}$ & Transversal \\
\hline $\begin{array}{l}\text { Huf, } \\
2000\end{array}$ & Brasil & $\begin{array}{l}\text { Se entrevistó a } 634 \text { mujeres mayores de } 60 \text { años que residían en centros } \\
\text { de adultos mayores en Río de Janeiro, entre mayo de } 1992 \text { y diciembre } \\
\text { de } 1995\end{array}$ & Transversal \\
\hline $\begin{array}{l}\text { Lima, } \\
2008\end{array}$ & Brasil & $\begin{array}{l}\text { Se entrevistaron } 1.023 \text { personas, mayores de } 14 \text { años, de la ciudad de } \\
\text { Botucatu, Brasil, entre los años } 2001 \text { y 2002. De ellos, } 439 \text { correspondieron } \\
\text { a sujetos mayores de } 60 \text { años }\end{array}$ & Transversal \\
\hline $\begin{array}{l}\text { Mendoza, } \\
2005\end{array}$ & Brasil & $\begin{array}{l}\text { Se analizaron } 1.336 \text { registros de pacientes atentidos en los últimos } 3 \text { meses } \\
\text { en Centro de Salud Escuela de la Facultad de Medicina de Ribeirao Preto, } \\
\text { de la Universidad de São Paulo. Se excluyeron pacientes no entrevistables } \\
\text { (con psicosis o Esquizofrenia). } 170 \text { pacientes eran mujeres con edad mayor } \\
\text { a } 60 \text { años, de las cuales } 72 \text { consumían BZD. Se entrevistaron a } 18 \text { de estas } \\
\text { pacientes }\end{array}$ & $\begin{array}{l}\text { Transversal/ } \\
\text { Cualitativo }\end{array}$ \\
\hline $\begin{array}{l}\text { Mendoza, } \\
2008\end{array}$ & Brasil & $\begin{array}{l}\text { Se analizaron } 1.336 \text { registros de pacientes atendidos en los últimos } 3 \\
\text { meses en Centro de Salud Escuela de la Facultad de Medicina de Ribeirao } \\
\text { Preto, de la Universidad de São Paulo. Se excluyeron pacientes no entrevis- } \\
\text { tables (con psicosis o Esquizofrenia). } 170 \text { pacientes eran mujeres con edad } \\
\text { mayor a } 60 \text { años, de las cuales } 72 \text { consumían BZD. Se entrevistaron a } 18 \\
\text { de estas pacientes }\end{array}$ & $\begin{array}{l}\text { Transversal/ } \\
\text { Cualitativo }\end{array}$ \\
\hline $\begin{array}{l}\text { Spanemberg, } \\
2011\end{array}$ & Brasil & $\begin{array}{l}\text { Se estudió el uso de drogas psicoactivas en } 504 \text { pacientes menores a } 60 \\
\text { años y } 71 \text { con edad igual o mayor a } 60 \text { años en la urgencia psiquiátrica en } \\
\text { el Hospital de San Lucas }\end{array}$ & Transversal \\
\hline
\end{tabular}


Tabla 1. Continuación

\begin{tabular}{|c|c|c|c|}
\hline $\begin{array}{l}\text { Autor, } \\
\text { año }\end{array}$ & País & Tipo de muestra y $n$ del estudio & $\begin{array}{c}\text { Diseño } \\
\text { experimental }\end{array}$ \\
\hline $\begin{array}{l}\text { Telles, } \\
2011\end{array}$ & Brasil & $\begin{array}{l}\text { De } 3.704 \text { pacientes atendidos en Consultorio de Minas Gerais, } 424 \text { tenían } \\
\text { edad mayor o igual a } 60 \text { años. De éstos, } 39 \text { eran usuarios de BZD, siendo } \\
\text { excluídos } 12 \text { por rechazo a participar o no haber podido ser contactados, } \\
\text { de manera que la muestra quedó constituida por } 27 \text { pacientes }\end{array}$ & Transversal \\
\hline $\begin{array}{l}\text { Busto, } \\
1996\end{array}$ & Chile & $\begin{array}{l}\text { Se entrevistaron } 1.500 \text { pacientes mayores de } 15 \text { años, en la ciudad de } \\
\text { Santiago, de los cuales } 186 \text { eran sujetos con edad igual o mayor a } 60 \text { años }\end{array}$ & Transversal \\
\hline $\begin{array}{l}\text { Gac, } \\
2003\end{array}$ & Chile & $\begin{array}{l}\text { Se estudiaron } 453 \text { pacientes institucionalizados y mayores de } 60 \text { años (383 } \\
\text { pacientes en residencias para autovalentes y } 70 \text { pacientes en hogares para } \\
\text { personas con deterioro cognitivo), en la ciudad de Santiago }\end{array}$ & Prospectivo \\
\hline $\begin{array}{l}\text { Galleguillos, } \\
2003\end{array}$ & Chile & $\begin{array}{l}\text { Se entrevistaron a } 188 \text { pacientes, mayores de } 14 \text { años, que fueron aten- } \\
\text { didos durante los días } 6 \text { y } 7 \text { de septiembre del año } 2000 \text {, en consultorios } \\
\text { de atención primaria en zonas urbanas de Santiago Centro y Recoleta y en } \\
\text { zonas rurales de Buin, Llay-Llay y Ancud. De la población entrevistada, } 45 \\
\text { sujetos tenían edad mayor a } 60 \text { años }\end{array}$ & Transversal \\
\hline $\begin{array}{l}\text { Sánchez, } \\
2010\end{array}$ & Cuba & $\begin{array}{l}\text { De manera aleatoria, se seleccionaron } 79 \text { de } 158 \text { pacientes mayores de } 60 \\
\text { años consumidores de BZD que se atendían en Consultorio Médico de la } \\
\text { Familia \#12, en La Habana }\end{array}$ & Transversal \\
\hline $\begin{array}{l}\text { Minaya, } \\
2009\end{array}$ & México & $\begin{array}{l}\text { Se estudiaron } 39 \text { pacientes, mayores de } 60 \text { años, con diagnóstico de } \\
\text { trastorno depresivo mayor o trastorno de ansiedad que acudieron conse- } \\
\text { cutivamente a Clínica de Psicogeriatría del Instituto Nacional de Psiquiatría } \\
\text { Ramón de la Fuente, en Ciudad de México }\end{array}$ & Transversal \\
\hline
\end{tabular}

\section{Tipos de BZD usadas en AM}

De los estudios revisados, 10 indicaron el tipo de BZD utilizada en poblaciones mayores de 60 años ${ }^{17,21,26,28,30,32,33,35,38,39}$, siendo las de mayor uso las de vida media larga. Entre los usuarios de BZD, la droga más frecuentemente utilizada fue el Diazepam, siendo la droga de elección en $77,8 \%{ }^{32}, 46,7 \%^{26}, 43 \%{ }^{38}, 37 \%{ }^{35}$ y $22,5 \%{ }^{17}$, de AM dependiendo del estudio.

Otras BZD frecuentemente utilizadas fueron el Clonazepam y el Lorazepam. La primera se encontró con frecuencias de uso de $68 \%{ }^{21}, 56,4 \%{ }^{39}$, $26 \%{ }^{35}$ y $12,6 \%{ }^{17}$, entre los usuarios de BZD. La segunda se utilizó con frecuencias de $7,8 \%{ }^{17}$, $25,6 \%{ }^{39}, 15,5 \%^{21}$ y $14,9 \%{ }^{30}$. Dentro de las otras BZD usadas se encontró al Bromazepam ${ }^{17,28,30,32,33}$ y Clordiazepóxido ${ }^{38}$, siendo la primera de éstas la más utilizada en tres de los cinco estudios en los cuales se menciona.

\section{Indicaciones para el uso de BZD en AM}

De los estudios revisados, seis indicaron las patologías por las que fueron utilizadas BZD en poblaciones mayores de 60 años, y sólo en cuatro de ellos fueron cuantificadas.

Dentro de las patologías tratadas con BZD, tres estudios refirieron su uso para manejo de síntomas ansiosos ${ }^{17,34,38}$, dos artículos para tratamiento de insomnio ${ }^{17,38}$ y dos trabajos para trastornos conductuales $^{22,34}$. Síntomas depresivos ${ }^{34}$, hipertensión $^{38}$, ideas suicidas ${ }^{34}$, convulsiones ${ }^{17}$ y síntomas psicóticos $^{34}$ fueron manejados con BZD en un estudio cada uno (Tabla 2).

\section{Consumo de BZD y asociación con variables sociodemográficas}

\section{a) Género}

De los estudios incluidos en esta revisión, diez artículos explicitaron la frecuencia de uso de BZD en AM por género $3,17,23,25,29,31,35,36,38,39$. Todos ellos indicaron un mayor porcentaje de uso de BZD en mujeres, con frecuencias que van desde $61 \%{ }^{3}$ hasta $92,3 \%{ }^{39}$.

Cuatro de estos trabajos realizaron un análisis multivariante en su población de estudio. 
Tabla 2. Porcentaje de AM tratados con BZD según indicación

\begin{tabular}{|c|c|c|c|c|}
\hline Indicación & $\begin{array}{c}\text { Rojas, } 2010 \\
\text { n (\%) } \\
\text { (pacientes } \\
\text { ambulatorios) }\end{array}$ & $\begin{array}{c}\text { Spanemberg, } 2011 \\
\text { n (\%) } \\
\text { (pacientes } \\
\text { ambulatorios) }\end{array}$ & $\begin{array}{c}\text { Sánchez, } 2010 \\
\text { n (\%) } \\
\text { (pacientes que } \\
\text { asistieron a servicio } \\
\text { de urgencias) }\end{array}$ & $\begin{array}{c}\text { Alvarenga, } 2008 \\
\text { n (\%) } \\
\text { (pacientes en } \\
\text { población de AM } \\
\text { no consultante) }\end{array}$ \\
\hline Síntomas depresivos & - & $22(31,4 \%)$ & - & - \\
\hline Hipertensión & - & - & $9(11,4 \%)$ & - \\
\hline Ideas suicidas & - & $16(22,9 \%)$ & - & - \\
\hline Ansiedad & - & $8(11,4 \%)$ & $43(54,5 \%)$ & $267(76,7 \%)$ \\
\hline Convulsiones & - & - & - & $44(12,6 \%)$ \\
\hline Insomnio & - & - & $23(29,1 \%)$ & $37(10,7 \%)$ \\
\hline $\begin{array}{l}\text { Manejo conductual } \\
\text { (agresividad, trastornos } \\
\text { cognitivos) }\end{array}$ & $88(100 \%)$ & $10(14,3 \%)$ & - & - \\
\hline Síntomas psicóticos & - & $6 \quad(8,6 \%)$ & - & - \\
\hline Otras & - & $8(11,4 \%)$ & $4 \quad(5 \%)$ & - \\
\hline
\end{tabular}

AM: Adultos mayores.

Alvarenga et al., luego de ajustar por variables como edad, estado civil, escolaridad e ingresos, concluyeron que el género femenino era la única variante que se asociaba en forma independiente a consumo de BZD (OR 1,93; IC 95\% = 1,51-2,46) ${ }^{17}$. En un nuevo estudio, el mismo autor evidenció una asociación positiva entre ser mujer y uso de BZD (OR 1,48, IC 95\% = 1,16-1,89) ${ }^{23}$. Lima et al., observaron una asociación similar (OR 2,2, IC $95 \%=1,1-4,5, \mathrm{p}=0,02)^{31}$, mientras que Brunoni et al., en un análisis bivariado, objetivaron una relación significativa entre género femenino y uso de BZD (OR 2,0, IC 95\% = 1,68-2,41, p < 0,001), la que dejó de ser significativa al analizarlo de forma multivariada ${ }^{25}$.

\section{b) Edad}

$\mathrm{Al}$ analizar la edad de los pacientes consumidores de BZD, se confirma su alto uso en AM.

Alvarenga et al., mostraron que la prevalencia de uso de BZD aumentó significativamente $(\mathrm{p}<0,001)$ con la edad en hombres, incrementándose de $11,6 \%$ en usuarios de 60-69 años, a $13,5 \%$ en usuarios de 70-79 años, hasta $28,6 \%$ en mayores de 79 años, siendo significativa la diferencia con $\mathrm{p}<0,001$. El consumo de BZD en mujeres en este mismo estudio no difirió en los distintos grupos etarios $(p=0,53)^{17}$. Por otro lado,
Sánchez y cols., que estudiaron 79 consumidores de BZD en mayores de 60 años, observaron que el grupo etario de mayor consumo fue el de 60-64 años, con 50,6\% del total, seguido del rango etario de 65-69 años, con 30,4\% $\%^{38}$.

Huf et al., cuya población correspondió solamente a mujeres AM, evidenciaron que las usuarias crónicas de BZD se concentraban en el rango etario de 70-74 años (44,7\%), seguido por el rango de 65-69 años $(40,4 \%)^{30}$. Por otra parte, Busto et al., concluyeron que los pacientes con edad igual o mayor a 60 años corresponden a 19,5\% del total de consumidores adultos de $\mathrm{BZD}^{36}$. Brunoni et al., obtuvieron como resultado que el riesgo de uso de BZD aumentaba con la edad, con un OR 2,97 (IC $95 \%=2-4,3, p<0,001)$ en análisis multivariado y OR 3,48 (IC 95\% = 2,54-4,76, p < 0,001) en análisis bivariado, para el rango de 65 a 75 años $^{25}$.

\section{c) Nivel socioeconómico (NSE)}

Tres de los estudios revisados analizaron la relación existente entre consumo de BZD en AM y NSE ${ }^{17,25,36}$. Ninguno de ellos encontró asociación significativa entre ambas variables. Sin embargo, Brunoni et al., reportaron que poseer seguro médico privado se relaciona en forma positiva con el uso de BZD (OR 2,09, IC 95\% = 1,6-2,6, $\mathrm{p}<0,001)^{25}$. 


\section{d) Escolaridad}

Cinco de los artículos incluidos analizaron la asociación entre nivel de escolaridad y consumo de BZD en AM, con resultados divergentes.

Alvarenga y Brunoni et al., no encontraron significativa relación ambas variables ${ }^{17,25}$. Por otro lado, los restantes tres estudios objetivaron una relación inversa entre nivel educacional y uso de BZD $^{30,35,36}$. Telles et al., mostraron que $66,6 \%$ de los consumidores tenía primer grado incompleto (grupo de escolaridad más bajo) ${ }^{35}$. Huf et al., señalaron que la mayoría de los consumidores AM de BZD (42,6\%) sólo tenía educación básica completa $^{30}$. Finalmente, Busto et al., concluyeron que el menor nivel educacional se asoció a mayor tasa de consumo de $\mathrm{BZD}^{36}$.

\section{Consumo de BZD y efectos adversos}

De los estudios revisados, siete señalaron efectos adversos en poblaciones mayores de 60 años en relación al uso de $\mathrm{BZD}^{24,26-28,30,37,39}$.

Dentro de los efectos adversos más reportados destacan las caídas, descritas en cuatro artículos $^{26-28,37}$. En el estudio de Gac et al., los pacientes AM que presentaron caídas usaban significativamente más BZD que el grupo control $(40,7 \% \text { versus } 29,4 \%)^{37}$. Por otro lado, Coutinho et al., objetivaron que el consumo de BZD en AM se asociaba a un mayor riesgo de fracturas por caídas (OR 2,27; IC 95\% = 1,20-4,31, p = 0,01) y mayor riesgo de fracturas graves (OR 2,09; IC $95 \%=1,08-4,05, \mathrm{p}=0,03)^{27}$. El mismo autor, en un estudio posterior constató que los AM que usaron BZD en las últimas 24 h poseían un riesgo significativamente mayor de caídas (OR 2,56; IC $95 \%=1,44-4,57)$ y de fracturas graves (OR 2,22; IC $95 \%=1,07-4,58)^{28}$. Finalmente, Chaimowicz et al., observaron una positiva asociación entre el consumo de BZD y caídas en AM $(\mathrm{p}=0,05)^{26}$.

Por otro lado, Huf et al., observaron que existe una asociación entre el uso a largo plazo de BZD y síntomas como cefalea crónica (OR 3,53; IC $95 \%=1,82-6,89)$ e insomnio (OR 8,87; IC $95 \%=2,53-31,06)^{30}$.

Sólo un estudio describió la dependencia a $\mathrm{BZD}$ en población de $\mathrm{AM}^{39}$. En este artículo, que incluye un total de 39 pacientes usuarios de BZD, $69,2 \%$ presentó síntomas de dependencia. De éstos, 92,6\% reportó haber tenido síntomas de abstinencia. De la misma forma, 59,35\% de los pacientes dependientes señalaron tolerancia a las
BZD y $88,9 \%$ indicó presentar síntomas residuales tras la reducción o cese del consumo de BZD. Además, se mencionó que la indicación médica inicial para el uso de BZD en los pacientes que desarrollaron dependencia fue el tratamiento de la ansiedad. Finalmente, Bicca et al., estudiaron el deterioro cognitivo asociado al uso de BZD, no encontrando asociación entre el uso prolongado de BZD y deterioro en la memoria $(\mathrm{p}=0,84)$, fluidez verbal $(\mathrm{p}=0,78)$ o la atención $(\mathrm{p}=0,23)^{24}$.

\section{Automedicación de BZD en AM}

De los estudios revisados, cuatro señalan automedicación en poblaciones mayores de 60 años $^{32,33,35,38}$. De ellos, sólo dos artículos hicieron un análisis cuantitativo, refiriendo una gran dispersión en la tasa de uso de BZD sin prescripción médica. Por un lado, Sánchez et al., constataron que $54,4 \%$ de los AM usuarios de BZD lo hacía de manera automedicada ${ }^{38}$, mientras que Telles et al., objetivaron una tasa de automedicación entre los AM usuarios de BZD de $11,1 \%{ }^{35}$. Mendoza et al., no refieren valores ${ }^{32,33}$.

\section{Discusión}

En los últimos años, extensa evidencia Europea y Norteamericana ha confirmado los riesgos del uso de BZD en AM, no sólo por su evidente riesgo de dependencia o efectos secundarios a corto plazo, sino que también por su asociación con fallas de memoria y demencia ${ }^{10,13}$.

La presente revisión entrega un panorama del grado de investigación en Latinoamérica en este tema.

Dentro de los principales resultados de los estudios revisados, se confirma la alta prevalencia de uso de BZD en AM, la cual llega hasta 49,5\% en población no consultante, $50,8 \%$ en pacientes hospitalizados y hasta $61,4 \%$ en atención primaria $^{21,34,36}$. Comparados con datos disponibles en la literatura internacional, las frecuencias de esta revisión son en general similares o superiores. A modo de ejemplo, dos estudios en población de AM no consultante mostraron prevalencias de consumo de BZD de $26,4 \%$ en España y $32 \%$ en Francia ${ }^{15,16}$. Es interesante la asociación reportada en algunos estudios y la variable edad ${ }^{17,25}$. Es hipotetizable que las BZD sean crecientemente utilizadas a medida que síntomas (e.g., alteraciones 
conductuales) asociados comúnmente a la vejez se hagan más aparentes.

Dentro de las BZD más consumidas por los AM destacan las de vida media larga (v.g. Diazepam) $)^{32}$, dato de relevancia, ya que si bien pueden poseer un potencial adictivo menor, poseen evidentes riesgos en AM debido a su mayor capacidad de producir efectos secundarios ${ }^{40}$. Destaca que las BZD son indicadas principalmente en el manejo de síntomas ansiosos e insomnio, y utilizadas con mayor frecuencia en mujeres, en congruencia con la prescripción de BZD en la población general ${ }^{1,6,41}$.

En relación a los efectos adversos, se confirma la asociación significativa que existe entre el uso de BZD en AM y efectos secundarios, e.g., caídas ${ }^{24-26,35}$. Sin embargo, pese a que está bien documentado que el consumo de BZD en AM se ha asociado a disfunción y deterioro cognitivo y aumento del riesgo de demencia, solamente se encontró un artículo que explora estos efectos adversos en población AM latinoamericana. Este estudio no reveló una relación significativa entre el uso de BZD y deterioro cognitivo en AM, lo que podría estar explicado por el bajo número de pacientes incluidos en el estudio y la dificultad para precisar el tiempo de uso de $\mathrm{BZD}^{24}$. El único trabajo que exploró el porcentaje de dependencia, reportó una alta prevalencia en usuarios $\mathrm{AM}^{39}$.

La presente revisión permite destacar las limitaciones de la información disponible. En primer lugar, el número de estudios que han abordado este tema en Latinoamérica es escaso. En efecto, más de la mitad de los estudios incluidos corresponden a un solo país, i.e., Brasil. La mayoría de los estudios son transversales y exploran características demográficas y aspectos generales de salud. De esa forma, gran parte de los resultados son descriptivos y no basados en hipótesis específicas que permitan un entendimiento mayor de este fenómeno. A diferencia de otra literatura ${ }^{42,43}$, en Latinoamérica no existen estudios de seguimiento a largo plazo con grandes poblaciones de usuarios de BZD.

Además, existe importante heterogeneidad respecto al lugar de residencia de la población de estudio (ambulatoria u hospitalizada). Con escasas excepciones ${ }^{23,24,26-28,37}$, los estudios incluidos no abordan detalladamente el impacto de las BZD en relación al riesgo de dependencia o su asociación a enfermedades o condiciones de mayor frecuencia en esta población de AM.
Finalmente, todos los estudios encontrados incluyeron como población de estudio a AM de ambos géneros o sólo mujeres, de manera que no existe información específica a nivel latinoamericano en relación al impacto del consumo de BZD en la salud de AM hombres.

En conclusión, los resultados obtenidos presentan ciertas similaridades respecto a la prevalencia, factores de riesgo y efectos adversos en relación al uso de BZD en los AM. Se confirma el alto uso de BZD en la población de AM latinoamericana, principalmente mujeres, con frecuente automedicación, y asociado a detrimentos de la salud.

Sin embargo, el escaso número de investigaciones en la región no permite la generalización de resultados, sugiriendo que la exploración del uso de BZD en AM se encuentra en etapas iniciales, $y$ denotando una necesidad imperiosa de mayor y más profundo estudio.

Surge entonces con fuerza la necesidad de nuevos y mejores estudios sobre la situación particular del consumo de BZD en Latinoamérica, en particular en países hispanoparlantes donde la evidencia actual es menor. El obtener información "local" permitirá crear mejores programas para la atención de $\mathrm{AM}$, tanto en relación a síntomas ansioso -depresivos como de aquellos síntomas conductuales derivados de la vejez, centrado en las necesidades concretas de nuestra población. Nuevas estrategias de diseños de investigación que consideren múltiples variables fundamentales en relación al uso de $\mathrm{BZD}$ son requeridas, tales como el tiempo, dosis y patrón de consumo; mayor tiempo de seguimiento; creación de estudios con cohortes conformadas por pacientes que inician uso de $\mathrm{BZD}$; y rigurosa determinación a la hora de objetivar demencias y deterioro cognitivo subclínico asociado al uso de estos fármacos.

Esta nueva información podrá ser usada también para una mayor educación a nuestra población tanto consultante como médica, para el manejo integral de la sintomatología del AM, considerando los riesgos a corto, mediano y largo plazo de nuestras decisiones clínicas.

\section{Referencias}

1. Cascade E, Kalali A. Use of Benzodiazepines in the Treatment of Anxiety. Psychiatry 2008; 5 (9): 21-2.

2. Donoghue J, Lader M. Usage of benzodiazepines: A review. Int J Psychiatry Clin Pract 2010; 14 (2): 78-87. 
3. Galleguillos T, Risco L, Garay JL, González M, Vogel M. Tendencia del uso de benzodiazepinas en una muestra de consultantes en atención primaria. Rev Med Chile 2003; 131 (5): 535-40.

4. McKernan RM, Rosahl TW, Reynolds DS, Sur C, Wafford KA, Atack JR, et al. Sedative but not anxiolytic properties of benzodiazepines are mediated by the GABAA receptor [alpha] 1 subtype. Nat Neurosci 2000; 3 (6): 587-92.

5. Olkkola KT, Ahonen J. Midazolam and Other Benzodiazepines. Handb Exp Pharmacol 2008; (182): 33560 .

6. Mant A, Mattick RP, de Burgh S, Donnelly N, Hall W. Benzodiazepine prescribing in general practice: dispelling some myths. Fam Pract 1995; 12 (1): 37-43.

7. Ashton $\mathrm{H}$. The diagnosis and management of benzodiazepine dependence. Curr Opin Psychiatry 2005; 18 (3): 249-55.

8. Cannon KT, Choi MM, Zúñiga MA. Potentially inappropriate medication use in elderly patients receiving home health care: A retrospective data analysis. Am J Geriatr Pharmacother 2006; 4 (2): 134-43.

9. Fick DM, Semla TP, Beizer J, Brandt N, Dombrowski R, DuBeau CE, et al. American Geriatrics Society 2015 Updated Beers Criteria for Potentially Inappropriate Medication Use in Older Adults. J Am Geriatr Soc 2015; 63 (11): 2227-46.

10. Billioti de Gage S, Bégaud B, Bazin F, Verdoux H, Dartigues JF, Pérez K, et al. Benzodiazepine use and risk of dementia: prospective population based study. BMJ 2012; 345: e6231.

11. Curran HV. Tranquillising memories: A review of the effects of benzodiazepines on human memory. Biol Psychol 1986; 23 (2): 179-213.

12. Park H, Satoh H, Miki A, Urushihara H, Sawada Y. Medications associated with falls in older people: systematic review of publications from a recent 5 -year period. Eur J Clin Pharmacol 2015; 71 (12): 1429-40.

13. Stewart SA. The effects of benzodiazepines on cognition. J Clin Psychiatry 2005; 66 Suppl 2: 9-13.

14. Beland SG, Preville M, Dubois MF, Lorrain D, Grenier $\mathrm{S}$, Voyer $\mathrm{P}$, et al. Benzodiazepine use and quality of sleep in the community-dwelling elderly population. Aging Ment Health 2010; 14 (7): 843-50.

15. Carrasco-Garrido P, Jiménez-García R, Astasio-Arbiza P, Ortega-Molina P, de Miguel AG. Psychotropics use in the Spanish elderly: predictors and evolution between years 1993 and 2003. Pharmacoepidemiol Drug Saf 2007; 16 (4): 449-57.

16. Fourrier A, Letenneur L, Dartigues JF, Moore N, Bégaud B. Benzodiazepine use in an elderly community-dwe- lling population. Eur J Clin Pharmacol 2001; 57 (5): 419-25.

17. Alvarenga JM, Loyola Filho AI, Firmo JO, Lima-Costa MF, Uchoa E. Prevalence and sociodemographic characteristics associated with benzodiazepines use among community dwelling older adults: the Bambuí Health and Aging Study (BHAS). Rev Bras Psiquiatr 2008; 30 (1): 7-11.

18. Peláez M, Wong RL. Vejez y recursos sociales en América Latina. En: Monografías Humanitas Volumen 1: Envejecimiento. Barcelona, España: Editorial José Manuel Ribera Casado; 2004. p. 73-84.

19. Centro Latinoamericano y Caribeño de Demografía (CELADE). Los Adultos Mayores en América Latina y el Caribe: Datos e Indicadores. Santiago: Boletín informativo; $2002.78 \mathrm{p}$.

20. Arriagada L, Jirón M, Ruiz I. Uso de medicamentos en el adulto mayor. Rev Hosp Clin Univ Chile 2008; 19: 309-17.

21. Marzi M, Diruscio V, Núñez M, Pires M, Quaglia N. Análisis de la prescripción de medicamentos en una comunidad geriátrica Argentina. Rev Med Chile 2013; 141 (2): 194-201.

22. Rojas G, Serrano C, Dillon C, Bartoloni L, Iturry M, Allegri R. Usos y abusos de fármacos en pacientes con deterioro cognitivo. Rev Arg de Psiquiat 2010; (21): 1823.

23. Alvarenga JM, Loyola Filho AI, Firmo JO, Lima-Costa MF, Uchoa E. A population based study on health conditions associated with the use of benzodiazepines among older adults (The Bambui Health and Aging Study). Cad Saude Publica 2009; 25 (3): 605-12.

24. Bicca MG, Argimon IIL. Habilidades cognitivas e uso de benzodiazepínicos em idosas institucionalizadas. J Bras Psiquiatr 2008; 57 (2): 133-8.

25. Brunoni AR, Nunes MA, Figueiredo R, Barreto SM, da Fonseca Mde J, Lotufo PA, et al. Patterns of benzodiazepine and antidepressant use among middle-aged adults. The Brazilian longitudinal study of adult health (ELSA-Brasil. J Affect Disord 2013; 151 (1): 71-7.

26. Chaimowicz F, Ferreira Tde J, Miguel DF. Use of psychoactive drugs and related falls among older people living in a community in Brazil. Rev Saude Publica 2000; 34 (6): 631-5.

27. Coutinho ESF, Da Silva SD. Medication as a risk factor for falls resulting in severe fractures in the elderly. Cad Saude Publica 2002; (18): 1359-66.

28. Coutinho ES, Fletcher A, Bloch KV, Rodrigues LC. Risk factors for falls with severe fracture in elderly people living in a middle-income country: a case control study. BMC Geriatr 2008; 8: 21. 
29. Gorzoni ML, Fabbri RMA, Pires SL. Medicamentos potencialmente inapropriados para idosos. Rev Assoc Med Bras 2012; 58 (4): 442-6.

30. Huf G, Lopes C, Rozenfeld S. Long-term benzodiazepine use in women at a daycare center for older people. Cad Saude Publica 2000; 16 (2): 351-62.

31. Lima MC, Menezes PR, Carandina L, Cesar CL, Barros $\mathrm{MB}$, Goldbaum M. [Common mental disorders and the use of psychoactive drugs: the impact of socioeconomic conditions]. Rev Saude Publica 2008; 42 (4): 717-23.

32. Mendonça RT, Carvalho ACD. O papel de mulheres idosas consumidoras de calmantes alopáticos na popularização do uso destes medicamentos. Rev Latinoam Enferm 2005; 13: 1207-12.

33. Mendonça RT, Carvalho ACD, Vieira EM, Adorno RCF. Medicalização de Mulheres Idosas e Interação com Consumo de Calmantes. Saúde Soc São Paulo 2008; 17 (2): 95-106.

34. Spanemberg L, Nogueira EL, da Silva CT, Dargél AA, Menezes FS, Cataldo Neto A. High prevalence and prescription of benzodiazepines for elderly: data from psychiatric consultation to patients from an emergency room of a general hospital. Gen Hosp Psychiatry 2011; 33 (1): 45-50.

35. Telles Filho PCP, Chagas AR, Pinheiro MLP, Lima AMJ, Durão MAS. Utilização de benzodiazepínicos por idosos de uma estratégia de saúde da família: implicações para enfermagem. Esc Anna Ner 2011; 15 (3): 581-6.

36. Busto UE, Ruiz I, Busto M, Gacitúa A. Benzodiazepine Use in Chile: Impact of Availability on Use, Abuse, and
Dependence. J Clin Psychopharmacol 1996; 16 (5): 363 72.

37. Gac H, Marín PP, Castro S, Hoyl T, Valenzuela E. Caídas en adultos mayores institucionalizados: descripción y evaluación geriátrica. Rev Med Chile 2003; 131 (8): 887-94.

38. Sánchez L, Hernández F. Consumo de benzodiazepinas en pacientes geriátricos del Consultorio \# 12, Policlínico "Campo Florido". Rev Cubana Farm 2010; 44 (3): 34653.

39. Minaya $\mathrm{O}$, Ugalde $\mathrm{O}$, Fresan A. Uso inapropiado de fármacos de prescripción: dependencia a benzodiazepinas en adultos mayores. Salud Mental 2009; 32: 405-11.

40. Rollason V, Vogt N. Reduction of polypharmacy in the elderly: a systematic review of the role of the pharmacist. Drugs Aging 2003; 20 (11): 817-32.

41. Boyd A, Van de Velde S, Pivette M, Ten Have M, Florescu S, O'Neill S, et al. Gender differences in psychotropic use across Europe: Results from a large cross-sectional, population-based study. Eur Psychiatry 2015; 30 (6): 778-88.

42. Vasudev A, Shariff SZ, Liu K, Burhan AM, Herrmann N, Leonard S, et al. Trends in Psychotropic Dispensing Among Older Adults with Dementia Living in LongTerm Care Facilities: 2004-2013. Am J Geriatr Psychiatry 2015; 23 (12): 1259-69.

43. Stephenson CP, Karanges E, McGregor IS. Trends in the utilisation of psychotropic medications in Australia from 2000 to 2011. Aust N Z J Psychiatry 2013; 47 (1): 74-87. 